\title{
Conservação e qualidade pós-colheita de quiabo sob diferentes tempera- turas e formas de armazenamento
}

\author{
Wagner F da Mota ${ }^{1}$; Fernando Luiz Finger²; Paulo Roberto Cecon ${ }^{4}$; Derly José H da Silva ${ }^{2}$; Paulo César \\ Corrêa $^{3}$; Lúcia P Firme ${ }^{2}$; Gisele P Mizobutsi ${ }^{1}$ \\ 'UNIMONTES-Depto. Ciências Agrárias, 39440-000 Janaúba-MG; ${ }^{2}$ UFV-Depto. Fitotecnia, 36571-000 Viçosa-MG; ${ }^{3}$ UFV-Depto. Eng. \\ Agrícola, 36571-000 Viçosa-MG; ${ }^{4}$ UFV-Depto. Informática, 36571-000 Viçosa-MG; wagner.mota@unimontes.br
}

\section{RESUMO}

O trabalho teve como objetivo avaliar a influência do filme plástico de PVC e da temperatura de armazenamento na conservação pós-colheita de frutos de cultivares de quiabo. O experimento foi organizado em delineamento de blocos casualizados, em esquema de parcelas subsubdivididas, tendo nas parcelas as duas temperaturas ( 5 e $10{ }^{\circ} \mathrm{C}$ ), nas subparcelas um fatorial 2 (sem e com PVC) x 4 (cultivares Amarelinho, Red Velvet, Star of David e Mammoth Spinless) e nas subsubparcelas os seis períodos de amostragem, com quatro blocos. A temperatura de $10^{\circ} \mathrm{C}$ e o uso do filme de PVC foram mais eficientes no controle da perda de massa da matéria fresca. O filme foi mais eficiente na manutenção de maior teor relativo de água no pericarpo dos frutos armazenados a 5 e $10^{\circ} \mathrm{C}$. A cv. Amarelinho perdeu menos massa da matéria fresca e manteve maior teor relativo de água. Os frutos armazenados a $5^{\circ} \mathrm{C}$ apresentaram maiores teores de vitamina $\mathrm{C}$, com menor perda de vitamina $\mathrm{C}$ nas cvs. Mammoth Spinless e Star of David. Observou-se maior incidência de injúria por frio e escurecimento nos frutos armazenados sem PVC e a $5^{\circ} \mathrm{C}$. A cv. Amarelinho apresentou melhor conservação pós-colheita com temperatura de $10^{\circ} \mathrm{C}$ e o uso da embalagem de PVC.

Palavras-chave: Abelmoschus esculentus, armazenamento, qualidade.

\begin{abstract}
Postharvest conservation and quality of okra submitted to different temperatures and storage forms

This work had the goal to evaluate the influence of PVC film and temperature on the postharvest storage life in four cultivars of okra. The treatments were displayed in randomized complete blocks, in split-split-plot design, where the parcels were the temperatures of 5 and $10^{\circ} \mathrm{C}$, and in the sub parcels a 2 (control and PVC wrapped fruits) x 4 (cultivars Amarelinho, Red Velvet, Star of David and Mammoth Spinless) factorial, and in the sub-sub parcels six sampling moments, containing four blocks. Storage at $10^{\circ} \mathrm{C}$ and wrapping the fruits with PVC film improved the control of fresh mass loss. The film was more efficient in maintaining higher water content in the fruit pericarp at 5 or $10^{\circ} \mathrm{C}$. The cultivar Amarelinho lost less fresh mass and maintained higher water content. Fruits stored at $5^{\circ} \mathrm{C}$ had higher vitamin C content. The cultivars Mammoth Spinless and Star of David showed lower losses of vitamin C. Cultivar Mammoth Spinless had the highest content of chlorophyll and Amarelinho the lowest. In general the development of chilling and browning was higher in fruits without PVC film at $5^{\circ} \mathrm{C}$. The cultivar Amarelinho had better postharvest conservation at $10^{\circ} \mathrm{C}$ and using PVC film.
\end{abstract}

Keywords: Abelmoschus esculentus, storage, quality.

(Recebido para publicação em 27 de fevereiro de 2009; aceito em 18 de janeiro de 2010) (Received on February 27, 2009; accepted on January 18, 2010)

$\mathrm{O}$ riginário da África, o quiabeiro (Abelmoschus esculentus) é uma hortaliça cultivada na África, Índia, Ásia, Estados Unidos, Turquia e Austrália (Duzyaman, 1997). No Brasil as condições para o seu cultivo são excelentes, principalmente no que diz respeito ao clima (Mota et al., 2000).

A possibilidade de exportação para países europeus que possuem comunidades apreciadoras do fruto surge como ótimo investimento. No entanto, é necessário produzir mais e com a qualidade exigida pelo mercado europeu. Em janeiro de 1999, houve uma primeira exportação de frutos de quiabo fresco para a França. Porém, não houve remessas posteriores à Europa, visto que as cultivares mais plantadas no Brasil, Amarelinho e Santa Cruz, não satisfazem as exigências do mercado francês quanto ao formato, tamanho, cor e qualidade do fruto.

As hortaliças são muito perecíveis e continuam o metabolismo respiratório após a colheita (Fonseca et al., 2000; Lee \& Kader, 2000). Por isso o período de conservação pós-colheita do quiabo é muito curto, principalmente em condições de armazenamento sob temperaturas altas e baixa umidade relativa, que aceleram a perda de água, depreciando o valor comercial dos frutos para o consumo in natura (Finger et al., 2008).

A temperatura e umidade relativa são importantes fatores que devem ser controlados para manutenção da qualidade como, aparência, textura, valor nutricional e flavor durante a armazenagem (Paull, 1999; Lee \& Kader, 2000). Assim, recomenda-se armazenar hortaliças em temperaturas mínimas que condicionem máxima conservação pós-colheita. Segundo Paull (1999), $90 \%$ dos produtos que deveriam ser armazenados à temperatura de $4^{\circ} \mathrm{C}$ ou menos são armazenados acima da faixa recomendada.

Temperaturas de armazenagem entre 5 e $15^{\circ} \mathrm{C}$ ocasionam injúrias por frio e escurecimento enzimático interno e externo em muitas espécies tropicais e subtropicais (Fernández-Trujilio et al., 1998a). No entanto, os frutos de feijão- 
vagem, pepino, quiabo, pimentão, abóbora e tomate, apesar de serem sensíveis à injúria por frio, toleram temperaturas um pouco mais baixas, permitindo que se faça o armazenamento em temperaturas entre 8 e $12^{\circ} \mathrm{C}$ (Pantástico et al., 1975). Estas desordens ocorrem em armazenamento prolongado, principalmente após retirada dos produtos da refrigeração (Fernández-Trujilio et al., 1998b). Contudo, outros atributos de qualidade, como textura, vitamina $\mathrm{C}$, flavor e aroma podem ser perdidos antes das mudanças externas serem observadas (Paull, 1999). Em quiabo, normalmente após três dias de armazenagem entre 1 e $6^{\circ} \mathrm{C}$, há descoloração do cálice, depressão da superfície dos frutos e as sementes tornam-se marrons (Tamura \& Minamide, 1984). Estes sintomas, aliados a manchas deprimidas de coloração escura, alterações metabólicas, amadurecimento, murchamento, perda de sabor e apodrecimento, caracterizam os sintomas de injúria por frio, chamado de chilling (Wang, 1989).

Uma das técnicas utilizadas para reduzir a incidência do chilling é a atmosfera modificada (Saltveit, 2000) pelo emprego de filme plástico, redução do teor de $\mathrm{O}_{2}$ e elevação do teor de $\mathrm{CO}_{2}$ complementados da refrigeração no armazenamento (Kader, 1995). Neste sistema as mudanças bioquímicas e a produção de etileno são reduzidos, retardando o amaciamento e a senescência (Pariasca et al., 2001). Ainda, injúrias físicas e fisiológicas são reduzidas, como a injúria por frio, prevenindo a perda de água (Lammertyn et al., 2000; Pesis et al., 2000).

No presente trabalho objetivou-se avaliar a influência de temperaturas e formas de armazenamento sobre a conservação e qualidade pós-colheita de quiabo.

\section{MATERIAL E MÉTODOS}

Os frutos de quiabo foram colhidos no campus da UFV, em Viçosa-MG, no ponto de colheita comercial, apresentando-se os frutos tenros, aproximadamente aos quatro dias após a antese e 60 dias após o plantio. Após a colheita os frutos foram acondicionados em caixas plásticas, e em seguida transportados para o Laboratório de Pós-Colheita, onde foram selecionados.

Os frutos foram armazenados em câmaras de refrigeração com umidade relativa de $95 \%$, monitoradas com termohigrógrafo. $\mathrm{O}$ experimento foi organizado em delineamento experimental de blocos casualizados, no esquema de parcelas subsubdivididas, tendo nas parcelas duas temperaturas $\left(5\right.$ e $\left.10^{\circ} \mathrm{C}\right)$, nas subparcelas um fatorial $2 \times 4$, ou seja, embalagens sem e com PVC (14 $\mu \mathrm{m}$ de espessura) e quatro cultivares (Amarelinho, Red Velvet, Star of David e Mammoth Spinless) e nas subsubparcelas seis períodos de amostragem com quatro blocos, sendo a unidade experimental constituída por 4 frutos.

As características físicas e químicas avaliadas em intervalos de três dias foram: Perda de massa da matéria fresca do fruto: a diferença de massa entre as avaliações foi acumulada durante a evolução do experimento e o resultado de perda de massa da matéria fresca em relação à massa inicial do fruto foi expresso em porcentagem; Teor relativo de água: foi avaliado conforme metodologia descrita por Catsky (1974), com o emprego da equação citada por Weatherley (1950):

$$
\Phi=\frac{F-W}{T-W} X 100
$$

em que $\Phi$ é o teor relativo de água; $\mathrm{F}$ a massa da matéria fresca; $\mathrm{W}$, a massa da matéria seca; e T, a massa da matéria túrgida. Foram retirados discos de 7 $\mathrm{mm}$ de diâmetro do pericarpo do quiabo e pesados. Os discos foram colocados em espumas saturadas de água por sete horas (tempo necessário para estabilização da massa) e pesados, obtendo-se a massa da matéria túrgida. Em seguida, foi obtida a massa da matéria seca por secagem em estufa a $70^{\circ} \mathrm{C}$ por 48 a 72 horas até atingir massa constante; Teor de clorofila: para avaliação da clorofila $\mathrm{a}, \mathrm{b}$ e total, foram retirados $3 \mathrm{~g}$ de massa da matéria fresca da parte mediana dos frutos, aos quais foram acrescentados 10 $\mathrm{mg}$ de sulfato de magnésio e $30 \mathrm{ml}$ de acetona 80\% (v/v) (Finger et al., 2008). Após a homogeinização, a suspensão foi filtrada, aferida num balão volumétrico de $50 \mathrm{~mL}$, sendo os teores de clorofila determinados espectrofotometricamente pelo método de Arnon (1949) nos comprimentos de onda de 645 e $663 \mathrm{~nm}$, expressando-se em $\mu / g$; Teor de vitamina $\mathbf{C}$ : determinado pela técnica recomendada pelo Instituto Adolfo Lutz (1985), e o resultado expresso em mg de ácido ascórbico por $100 \mathrm{~g}$ de matéria fresca; Aparência visual: utilizou-se uma escala (Finger et al., 2008), onde as notas variam de 0 a 4 , conforme o estado de conservação desses frutos. Foram avaliadas as lesões de injúria por frio e o aparecimento de manchas. As lesões de injúria por frio, relativas ao grau de severidade de ocorrência, foram avaliadas mediante as notas: 0 - sem injúria, ausência de ponto de injúria; 1 - levemente injuriados, menos de cinco pontos; 2 - moderadamente injuriados, 5 a 10 pontos; 3 - extremamente injuriados, de 10 a 15 pontos; e 4 - completamente injuriados, com mais de 15 pontos maiores e por todo o fruto; Escurecimento do pericarpo: a presença de manchas de descoloração foi avaliada com o auxílio das notas: 0 - sem escurecimento, ausência de manchas escuras; 1 - levemente escurecidos, com pequenas manchas ou levemente escurecidos; 2 - moderadamente escurecidos, com manchas maiores; 3 - extremamente escurecidos, com manchas distribuídas por todo o fruto; e 4 - completamente escurecidos quando as manchas escuras ocupavam mais de $50 \%$ do fruto.

Os dados foram interpretados por meio de análise de variância e de regressão com a utilização do programa SAEG (1997). As médias dos fatores qualitativos foram comparadas, utilizando-se o teste de Tukey a 5\% de probabilidade. Para os fatores quantitativos, os modelos foram escolhidos com base na significância dos coeficientes de regressão, a 5\% de probabilidade, no coeficiente de determinação e no potencial para explicar o fenômeno biológico em questão.

\section{RESULTADOS E DISCUSSÃO}

Houve aumento linear de 1,25 e $1,55 \%$ de perda de massa da matéria fresca por dia nas temperaturas de 5 e $10^{\circ} \mathrm{C}$ respectivamente, em todas as cultivares (Figura 1C). Os frutos embalados a $10^{\circ} \mathrm{C}$ perderam mais massa 


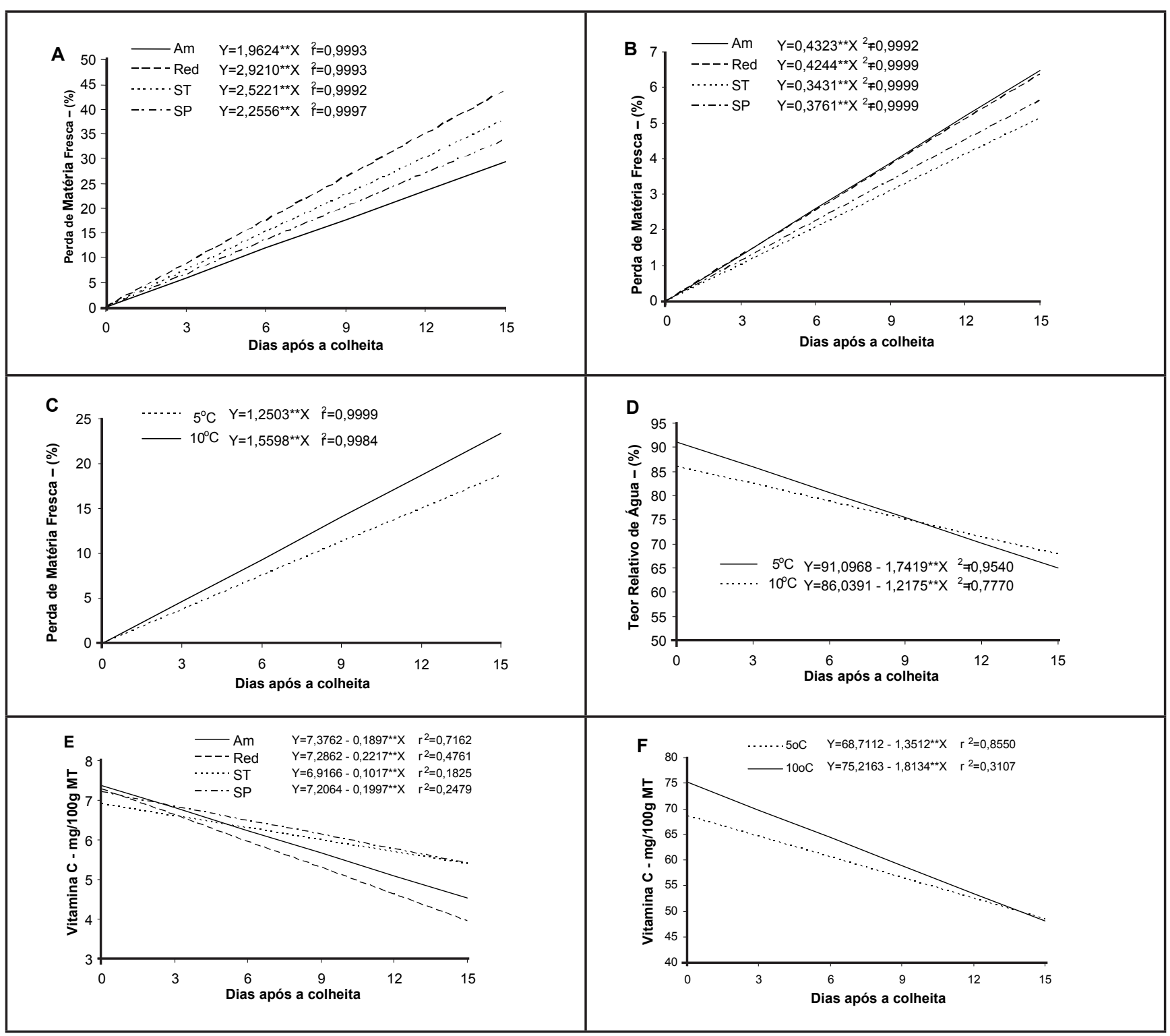

Figura 1. Perda da massa fresca (\%), teor relativo de água e teor de vitamina $\mathrm{C}$ em frutos de quiabo das cvs. Amarelinho (Am), Red Velvet (Red), Star of David (ST) e Mammoth Spinless (SP) (fresh matter loss (\%), relative water content and vitamin C content in fruits of okra cvs. Amarelinho (Am), Red Velvet (Red), Star of David (ST) and Mammoth Spinless (SP)); 1A - Perda da massa fresca em frutos sem PVC (\%) (Fresh matter loss in fruits without PVC (\%)); 1B - Perda de massa de matéria fresca em frutos com PVC (Fresh matter loss in fruits with PVC (\%)); $1 \mathrm{C}$ - Perda de massa de matéria fresca a 5 e $10^{\circ} \mathrm{C}(\%)$ (Fresh matter loss in fruits at 5 and $\left.10^{\circ} \mathrm{C}(\%)\right)$; $1 \mathrm{D}-\mathrm{Teor}$ relativo de água em frutos sem PVC a 5 e $10^{\circ} \mathrm{C}$ (relative water content in fruits stored at 5 and $10^{\circ} \mathrm{C}$ without PVC); $1 \mathrm{E}-\mathrm{Teor}$ de vitamina $\mathrm{C}$ (vitamin $\mathrm{C}$ content); $1 \mathrm{~F}$ - Teor de vitamina $\mathrm{C}$ a 5 e $10^{\circ} \mathrm{C}$ (content of vitamin $\mathrm{C}$ in fruits stored at 5 and $10^{\circ} \mathrm{C}$ ). Janaúba, UNIMONTES, 2008.

da matéria fresca. Esses resultados são concordantes com os de Finger et al. (2008), Carvalho (2002) e Modolo et al. (2000), que também observaram maior perda de massa para os frutos armazenados a $10^{\circ} \mathrm{C}$. A maior parte da perda de massa foi devida à perda de água ocasionada pela diferença de pressão de vapor de água entre os ambientes interno e externo do fruto (Taiz \& Zeiger, 1991). A redução da temperatura reduz a atividade metabólica e a troca gasosa com o meio reduzindo a respiração e aumentando a conservação pós-colheita de produtos hortícolas (Bower, 1998; Carvalho, 2002).

A perda de massa da matéria fresca aumentou durante o armazenamento dos frutos de todas as cultivares, armazenados sem e com PVC (Figura 1A, 1B), sendo muito superior nos frutos das cultivares armazenadas sem PVC. Essa maior eficiência no uso da embalagem com PVC também foi verificada por Ta- mura \& Minamide (1984), por Modolo et al. (2000) e por Finger et al. (2008). A cv. Amarelinho apresentou redução da perda de massa da matéria fresca em aproximadamente $20 \%$ com o uso de PVC em relação aos frutos acondicionados sem este filme polimérico (Finger et al., 2008).

A embalagem de PVC agiu passivamente no controle da perda de massa da matéria fresca, pois a barreira física imposta pelo filme condicionou níveis 


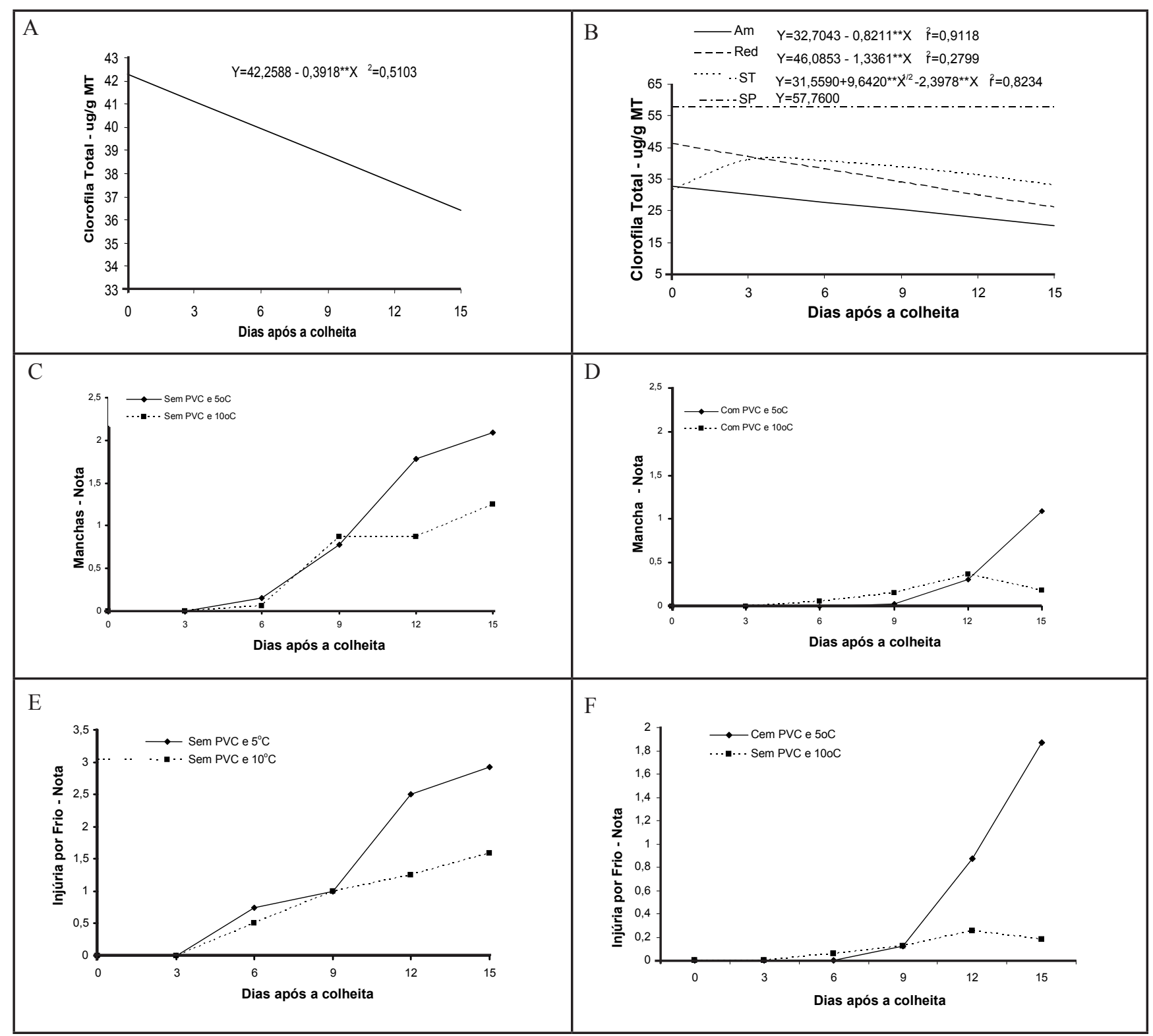

Figura 2. Teores de clorofila, manchas escuras e de injúria por frio em frutos de quiabo das cvs. Amarelinho (Am), Red Velvet (Red), Star of David (ST) e Mammoth Spinless (SP) (chlorophyll content, browning and chilling symptoms in okra fruits cvs. Amarelinho (Am), Red Velvet (Red), Star of David (ST) and Mammoth Spinless (SP)); 2A - Teores de clorofila total dos frutos armazenados (total chlorophyll content in stored fruits); $2 \mathrm{~B}$ - Teores de clorofila total das cultivares de quiabo em função dos dias após a colheita (total chlorophyll content in cultivars of okra fruits stored at 5 and $10^{\circ} \mathrm{C}$ ); $2 \mathrm{C}$ - Desenvolvimento de manchas escuras sem PVC a 5 e $10^{\circ} \mathrm{C}$ ao longo do período de armazenamento (development of browning in fruits stored without PVC stored at 5 and $10^{\circ} \mathrm{C}$ ); $2 \mathrm{D}-$ Desenvolvimento de manchas escuras em frutos armazenados com PVC a 5 e $10^{\circ} \mathrm{C}$ (development of browning in fruits stored with PVC at 5 and $10^{\circ} \mathrm{C}$ ); $2 \mathrm{E}-\mathrm{Desenvolvimento}$ de injúria por frio em frutos armazenados sem PVC a 5 e $10^{\circ} \mathrm{C}$ (development of chilling symtoms in fruits stored without PVC at 5 and $10^{\circ} \mathrm{C}$ ); $2 \mathrm{~F}$ - Desenvolvimento de sintomas de injúria por frio em frutos armazenados com PVC a 5 e $10^{\circ} \mathrm{C}$ (development of chilling injury symptoms in fruit stored with PVC at 5 and $10^{\circ} \mathrm{C}$ ). Janaúba, UNIMONTES, 2008.

elevados de umidade dentro da embalagem, havendo redução do gradiente de pressão de vapor de água entre os frutos e a atmosfera interna da embalagem, reduzindo então, a perda de massa da matéria fresca (Ben-Yehoshua, 1985; Fonseca et al., 2000; Pesis et al., 2000). Com relação aos frutos das cvs. não embaladas, observou-se diferenças entre os materiais, sendo que os frutos da cv. Red Velvet apresentaram maior perda de massa da matéria fresca e os frutos das cvs. Amarelinho e Mammoth Spinless perderam menos.

Houve redução linear do teor relativo de água, ao longo do armazenamento, nos frutos armazenados sem PVC, nas temperaturas de 5 e $10^{\circ} \mathrm{C}$ (Figura 1D).
Contrariamente, nos frutos armazenados com PVC, o teor relativo de água manteve-se constante nas temperaturas de 10 e $5^{\circ} \mathrm{C}$, respectivamente, demonstrando maior eficiência. Nos frutos armazenados sem PVC, a cv. Amarelinho apresentou maior teor de água e as cvs. Red Velvet e Mammoth Spinless os menores teores (Tabela 1). A maior 


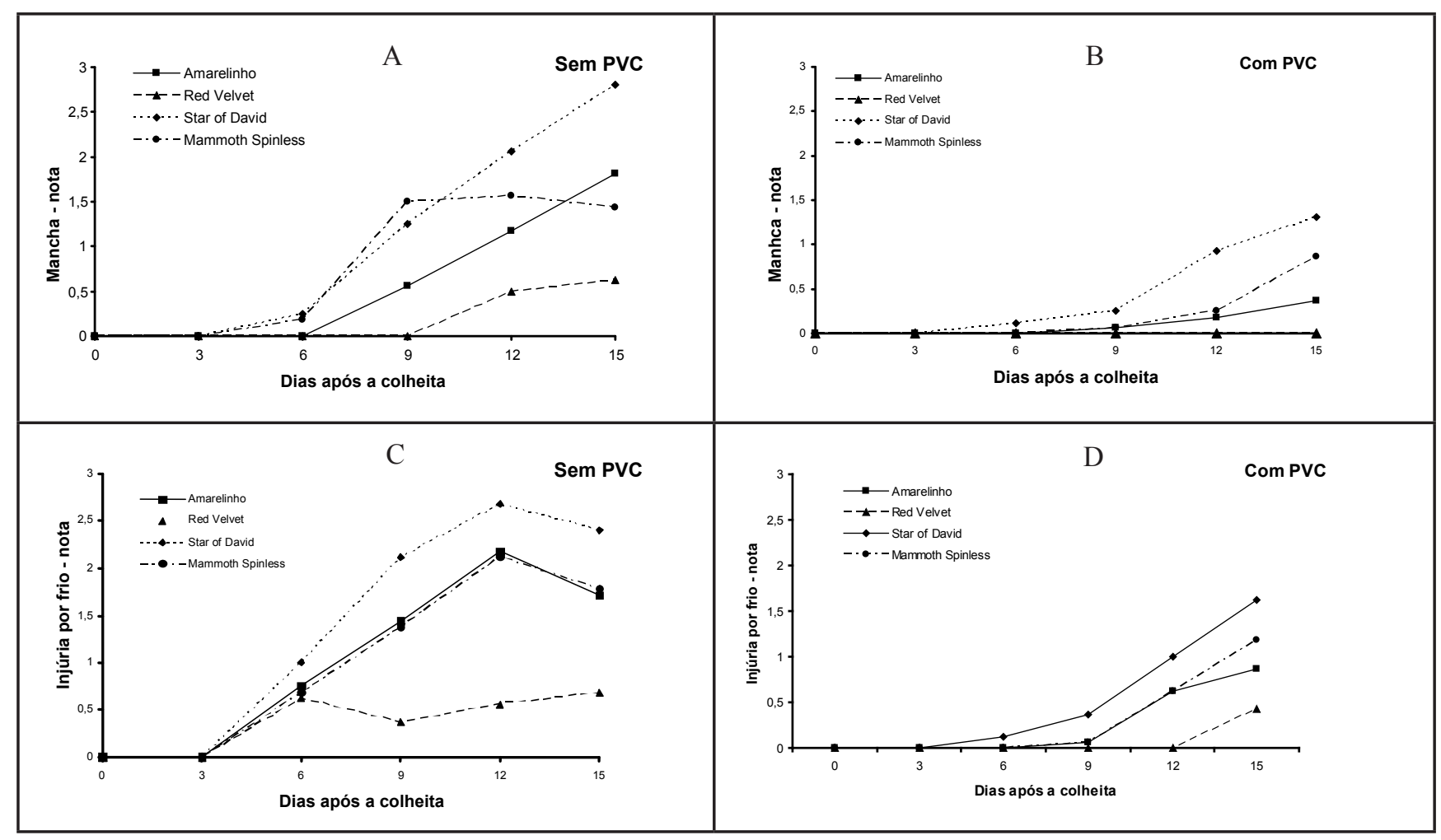

Figura 3. Desenvolvimento de manchas escuras e injúria por frio em frutos de quiabo cvs. Amarelinho (Am), Red Velvet (Red), Star of David (ST) e Mammoth Spinless (SP) armazenados a 5 e $10^{\circ} \mathrm{C}$ (development of browning and chilling symtoms in okra fruits cvs. Amarelinho (Am), Red Velvet (Red), Star of David (ST) and Mammoth Spinless (SP)); 3A - Manchas escuras em frutos sem PVC (browning in fruits of okra without PVC); 3B - Manchas escuras em frutos com PVC (browning in fruits of okra with PVC); 3C -Injúria por frio em frutos sem PVC (chilling symptoms in fruits of okra without PVC); 3D - Injúria por frio em frutos com PVC (chilling symptoms in fruits of okra stored with PVC). Janaúba, UNIMONTES, 2008.

eficiência relativa dos frutos armazenados com filme plástico também foi verificada por Finger et al. (2008), ao trabalhar com a cv. Amarelinho.

Adicionalmente à perda de água, ocorre aumento proporcional do teor de fibras em função da murcha dos frutos (Carvalho, 2002). Assim, a redução da perda de água durante o armazenamento, que foi atingida principalmente com o uso de frutos embalados com PVC, é fundamental para manter as qualidades aparente e nutricional (Burdon \& Clark, 2001).

Houve redução linear do teor de vitamina $\mathrm{C}$ nas temperaturas de 5 e $10^{\circ} \mathrm{C}$ (Figura 1F). Esta mesma tendência foi encontrada por Finger et al. (2008). No entanto, de acordo com Paull (1999) e Lee \& Kader (2000), as maiores perdas ocorreriam a $10^{\circ} \mathrm{C}$, pois a vitamina $\mathrm{C}$ é mais sensível à degradação quando as hortaliças estão sujeitas a temperaturas mais elevadas. Entretanto, as perdas de vitamina $C$ são maiores também em frutos sob injúria por frio, e a destruição do ácido ascórbico pode ocorrer antes do surgimento dos visíveis sintomas de chilling. A redução da temperatura de 10 para $5^{\circ} \mathrm{C}$ foi praticamente insignificante na manutenção de maiores teores de vitamina $\mathrm{C}$, e a redução provavelmente é devida à incidência de injúria por frio nos frutos armazenados a $5^{\circ} \mathrm{C}($ Lee \& Kader, 2000).

Foi observada redução linear do teor de vitamina $\mathrm{C}$ em todas as cvs. estudadas (Figura 1E). A cultivar Red Velvet teria vantagem comparativa, pois apresentou maior teor de vitamina $\mathrm{C}$ no dia " 0 ". Entretanto, foi a cultivar que apresentou maior perda desse nutriente ao longo do período de armazenamento. Por outro lado, as cvs. Mammoth Spinless e Star of David manifestaram as menores perdas ao longo do período de armazenamento.

Frutos de quiabo embalados com PVC apresentaram em média 6,32 $\mathrm{mg} / 100 \mathrm{~g}$ de vitamina $\mathrm{C}, 40 \mu \mathrm{g} / \mathrm{g}$ de clorofila total e $23,52 \mu \mathrm{g} / \mathrm{g}$ de clorofila "a", enquanto os frutos mantidos sem embalagem apresentaram 5,69 mg/100g, $37,67 \mu \mathrm{g} / \mathrm{g}$ e $22,04 \mu \mathrm{g} / \mathrm{g}$, respectivamente. As condições que favorecem menor perda de água e murchamento resultam em menor perda de vitamina (Lee \& Kader, 2000). Finger et al. (2008), ao armazenar frutos da cv. Amarelinho, observaram que os armazenados com PVC apresentaram teores médios de vitamina $C$ superiores nas temperaturas de 5 e $10^{\circ} \mathrm{C}$, respectivamente.

Em geral, observou-se redução dos teores de clorofila total ao longo do armazenamento (Figura 2A). Em quiabo, a perda de clorofila e de coloração verde são reflexos da senescência (Beaudry, 1999). Esta redução de clorofila total é mais elevada na cultivar Red Velvet (Figura 2B). Com relação à cv. Star of David, houve aumento do teor de clorofila total até o terceiro dia aproximadamente, porém deste dia em diante houve redução. Houve manutenção do elevado teor de clorofila total ao longo do período de armazenamento na $\mathrm{cv}$. Mammoth Spinless, mantendo maior 
Tabela 1. Valores médios do teor relativo de água dos frutos das cvs. de quiabeiro Amarelinho, Red Velvet, Star of David e Mammoth Spinless embalados sem e com PVC (average of relative water content in fruits of okra cultivars Amarelinho, Red Velvet, Star of David and Mammoth Spinless stored with and without PVC). Janaúba, UNIMONTES, 2008.

\begin{tabular}{lll}
\hline \multirow{2}{*}{ Cultivar } & \multicolumn{2}{c}{ Teor relativo de água (\%) } \\
\cline { 2 - 3 } & Sem PVC & Com PVC \\
\hline Amarelinho & $80,85 \mathrm{aB}$ & $92,25 \mathrm{aA}$ \\
Red Velvet & $75,41 \mathrm{bB}$ & $92,30 \mathrm{aA}$ \\
Star of David & $77,58 \mathrm{abB}$ & $93,28 \mathrm{aA}$ \\
Mammoth Spinless & $76,01 \mathrm{bB}$ & $92,33 \mathrm{aA}$ \\
\hline
\end{tabular}

As medias seguidas de pelo menos uma mesma letra maiúscula, nas colunas, e minúscula, nas linhas não diferem entre si, a 5\% de probabilidade, pelo teste de Tukey (means followed by the same upper case letter in the column, and lower case in the row do not differ from each other by Tukey test at $5 \%$ ).

teor em relação às outras cultivares.

Não foi observado efeito da atmosfera modificada nem da baixa temperatura no desverdecimento de frutos de quiabo, como observado por Pariasca et al. (2001) em que, ao realizar a combinação atmosfera modificada com baixa temperatura, houve redução da degradação de clorofila em ervilha. Provavelmente, este fato ocorreu em função da elevada permeabilidade a gases verificada nos filmes poliméricos de PVC (Robertson, 1993; Miller \& Krochta, 1997). Apesar de Finger et al. (2008) terem verificado eficiência no controle da perda de clorofila com o uso da atmosfera modificada, concluiu-se que controle maior é obtido com a redução da temperatura. Entretanto, Carvalho (2002) também verificou que temperaturas de 5 e $10^{\circ} \mathrm{C}$ não influenciaram nos teores de clorofila, mesmo estas temperaturas causando injúria por frio. Segundo Hakim et al. (1999), a destruição de clorofila acontece como resultado do chilling, que ocasiona peroxidação deteriorativa das células da membrana.

Observa-se pelas Figuras 2C e 2D que os frutos armazenados nas temperaturas de 5 e $10^{\circ} \mathrm{C}$, embalados ou não com PVC, mantiveram ótimo estado de conservação até três dias após a colheita, evidenciando ausência de sintomas de injúria por frio e escurecimento, obtendo portanto a nota " 0 ". Finger et al. (2008) verificaram esta eficiência até o segundo dia após o armazenamento ao trabalhar com a cv. Amarelinho nas mesmas temperaturas, prolongando-se a eficiência com a temperatura de $10^{\circ} \mathrm{C}$ até o $10^{\circ}$ dia.
Os frutos armazenados a $5^{\circ} \mathrm{C}$ com PVC mantiveram ótimo estado de conservação até o sexto dia. Observou-se, de maneira geral, maior incidência de injúria por frio e escurecimento nos frutos armazenados sem PVC. Com relação às temperaturas, a incidência foi idêntica até 3 dias após a colheita e destes pontos em diante os frutos armazenados a $5^{\circ} \mathrm{C}$ passaram a manifestar maior incidência de injúria e escurecimento. Essa maior eficiência dos frutos armazenados em filmes poliméricos no controle da injúria por frio, e maior incidência nos frutos armazenados a $5^{\circ} \mathrm{C}$, foi verificada por Della-Justina (1998), Carvalho (2002), Tamura \& Minamide (1984). Segundo Awad (1993), os sintomas de injúria por frio se tornam mais severos ainda quando os frutos são transferidos da temperatura injuriante para ambiente com temperaturas superiores, fato este que foi observado por Carvalho (2002).

Com relação às cultivares, foi verificado ótimo estado de conservação com ausência de injúria e escurecimento em todas as cultivares, sem ou com filme polimérico de PVC até 3 dias após a colheita (Figura 3). A cultivar Red Velvet, armazenada com PVC, manteve ótimo estado de conservação durante o armazenamento pós-colheita com o menor índice de escurecimento e injúria por frio. Frutos como maçã e melão também apresentam diferença varietal em relação ao chilling (Paull, 1999).

Tem sido demonstrado que elevados teores de $\mathrm{CO}_{2}$ e reduzidos de $\mathrm{O}_{2}$ são benéficos no alívio dos sintomas de chilling em produtos sensíveis a esse estresse, como lima, abacate, manga e pepino (Pesis et al., 2000; Ju et al., 2000), além de ter manifestado eficiência no presente experimento ao se usar filme de PVC. São relatados ainda o efeito do acúmulo de etanol e acetaldeído em frutos embalados em filmes não perfurados no alívio dos sintomas do chilling (Fernández-Trujilio et al., 1998b). Por outro lado, foi sugerido que a redução da transpiração tem efeito mais pronunciado na redução da injúria por frio (Hakim et al., 1999).

A temperatura de $10^{\circ} \mathrm{C}$ e o uso da embalagem de PVC foram mais eficientes na conservação e qualidade póscolheita de frutos de quiabo, com maior eficiência no controle da perda de matéria fresca do fruto, manutenção do teor relativo de água no pericarpo, menor incidência de injúria por frio e escurecimento. A cv. Amarelinho demonstrou bom estado de conservação com menor perda de matéria fresca e manutenção de maior teor relativo de água, enquanto a cv. Red Velvet apresentou, de maneira, geral menor conservação.

\section{REFERÊNCIAS}

ARNON DI. 1949. Copper enzyme in isolated chloroplasts polyphenoloxidase in Beta vulgaris. Plant Physiology 24: 1-15.

AWAD M. 1993. Fisiologia pós-colheita de frutos. São Paulo: Nobel. 114p.

BEAUDRY RM. 1999. Effect of $\mathrm{O}_{2}$ and $\mathrm{CO}_{2}$ partial pressure on selected phenomena affecting fruit and vegetable quality. Postharvest Biology and Technology 15: 293-303.

BEN-YEHOSHUA S. 1985. Individual sealpackaging of fruit and vegetables in plastic film - A new postharvest technique. HortScience 20: 32-37.

BOWER JH; JOBLING JJ; PATTERSON BD; RYAN DJ. 1998. A method for measuring the respiration rate and respiratory quotient of detached plant tissues. Postharvest Biology and Technology 13: 263-270.

BURDON J; CLARK C. 2001. Effect of postharvest water loss on 'Hayward' kiwifruit water status. Postharvest Biology and Technology 22: 215-225.

CARVALHO MJ. 2002. Injúria por frio, respiração e produção de etileno pós-colheita em quiabo (Abelmoschus esculentus (L.) Moench.). Viçosa: UFV. (Tese mestrado).

CATSKY J. 1974. Water content. In: SLAVIK B. (ed). Methods of studying plant water relations. Berlin: Springer-Verlag. p.121-31.

DUZYAMAN E. 1997. Okra: botany and horticulture. In: JANICK J. Horticultural Reviews p. 41-72.

FERNANDEZ-TRUJILIO JP; MARTINEZ JA; ARTÉS F. 1998a. Modified atmosphere 
packaging affects the incidence of cold storage disorders and keeps "flat" peach quality. Food Research International 31: 571-579.

FERNANDEZ-TRUJILIO JP; CANO A; ARTÉS F. 1998b. Physiological changes in peaches related to chilling injury and ripening. Postharvest Biology and Technology 13: 109-119.

FINGER FL; DELLA-JUSTINA ME; CASALI VWD; PUIATTI M. 2008. Temperature and modified atmosphere affect the quality of okra. Scientia Agricola 65: 360-364.

FONSECA SC; OLIVEIRA FAR; LINO IBM; BRECHT J; CHAU KV. 2000. Modelling $\mathrm{O}_{2}$ and $\mathrm{CO}_{2}$ exchange for development of perforation-mediated modified atmosphere packaging. Journal of Food Engineering 43: 9-15.

HAKIM A; PURVIS AC; MULLINIX BG. 1999. Differences in chilling sensitivity of cucumber varieties depends on storage temperature and the physiological disfunction evaluated. Postharvest Biology and Technology 17: 97-104.

INSTITUTO ADOLFO LUTZ. 1985. Normas analiticas do Instituto Adolfo Lutz; métodos químicos efísicos para análises de alimentos. São Paulo. 183p.

JU Z; DUAN Y; JU Z. 2000. Plant oil emulsion modifies internal atmosphere, delays fruit ripening, and inhibits internal browning in Chinese pears. Postharvest Biology and Technology 20: 243-250.

KADER AA. 1995. Regulation of fruit physiology by controlled/modified atmosphere. Acta
Horticulturae 398:59-67.

LAMMERTYN J; AERTS M; VERLINDEN BE; SCHOTSMANS W; NICOLAI BM. 2000. Logistic regression analysis of factors influencing core breakdown in 'Conference' pears. Postharvest Biology and Technology 20: 25-37.

LEE SK; KADER AA. 2000. Preharvest and postharvest factors influencing vitamin $\mathrm{C}$ content of horticultural crops. Postharvest Biology and Technology 20: 207-220.

MILLER KS; KROCHTA JM. 1997. Oxygen and aroma barrier properties of edible films: A review. Trends in Food Science \& Technology 8: 228-237.

MODOLO VA; KLUGE RA; JACOMINO AP. 2000. Pós-colheita de quiabos submetidos à refrigeração e embalagem plástica. Horticultura Brasileira 18: 298-299.

MOTA WF; FINGER FL; CASALI VWD. 2000. Olericultura: Melhoramento Genético do Quiabeiro. Viçosa: UFV. 144p.

PANTASTICO EB; CHATTOPADHYAY TK; SUBRAMANYAM H. 1975. Storage and commercial storage operations, in postharvest physiology, handling and utilization of tropical and subtropical fruits and vegetables. Westport: AVI. 314p.

PARIASCA JAT; MIYAZAKI T; HISAKA H; NAKAGAWA H; SATO T. 2001. Effect of modified atmosphere packaging (MAP) and controlled atmosphere (CA) storage on the quality of snow pea pods (Pisum sativum L. var. saccharatum). Postharvest Biology and Technology 21: 213-223.
PAULL RE. 1999. Effect of temperature and relative humidity on fresh commodity quality. Postharvest Biology and Technology 15: 263-277.

PESIS E; AHARONI D; AHARON Z; BEN-ARIE R; AHARONI N; FUCHS Y. 2000. Modified atmosphere and modified humidity packaging alleviates chilling injury symptoms in mango fruit. Postharvest Biology and Technology 19: 93-101.

ROBERTSON GL. 1993. Food Packaging. New York: Marcel Dekker. 676p.

SALTVEIT ME. 2000. Wound induced changes in phenolic metabolism and tissue browning are altered by heat shock. Postharvest Biology and Technology 15: 61-69.

TAIZ L; ZEIGER E. 1991. Plant physiology. Redwood: Benjamin/Cummings. 565p.

TAMURA J; MINAMIDE T. 1984. Harvesting maturity, handling, storage of okra pods. Bulletin University Osaka Prefect Series B 36: 87-97.

UNIVERSIDADE FEDERAL DE VIÇOSA - UFV. 1997. SAEG - Sistema de análises estatísticas e genéticas. Versão 7.1. Viçosa, MG: UFV. 150p. (Manual do usuário)

WANG, CY. 1989. Chilling injury of fruits and vegetables. Food Reviews International 5: 209-236.

WEATHERLEY PE. 1950. Studies in the water relations of cotton plant. I - The field measurement of water deficits in leaves. New Phytologist 49: 81-97. 